\title{
Cross-species inference of long non-coding RNAs greatly expands the ruminant transcriptome
}

\author{
Stephen J. Bush ${ }^{1,2^{*}}$, Charity Muriuki ${ }^{1}$, Mary E. B. McCulloch ${ }^{1}$, Iseabail L. Farquhar ${ }^{3}$, Emily L. Clark ${ }^{1 \dagger}$ \\ and David A. Hume $e^{1,4^{*}+}$
}

\begin{abstract}
Background: mRNA-like long non-coding RNAs (IncRNAs) are a significant component of mammalian transcriptomes, although most are expressed only at low levels, with high tissue-specificity and/or at specific developmental stages. Thus, in many cases IncRNA detection by RNA-sequencing (RNA-seq) is compromised by stochastic sampling. To account for this and create a catalogue of ruminant IncRNAs, we compared de novo assembled IncRNAs derived from large RNA-seq datasets in transcriptional atlas projects for sheep and goats with previous IncRNAs assembled in cattle and human. We then combined the novel IncRNAs with the sheep transcriptional atlas to identify co-regulated sets of protein-coding and non-coding loci.
\end{abstract}

Results: Few IncRNAs could be reproducibly assembled from a single dataset, even with deep sequencing of the same tissues from multiple animals. Furthermore, there was little sequence overlap between IncRNAs that were assembled from pooled RNA-seq data. We combined positional conservation (synteny) with cross-species mapping of candidate IncRNAs to identify a consensus set of ruminant IncRNAs and then used the RNA-seq data to demonstrate detectable and reproducible expression in each species. In sheep, 20 to 30\% of IncRNAs were located close to proteincoding genes with which they are strongly co-expressed, which is consistent with the evolutionary origin of some ncRNAs in enhancer sequences. Nevertheless, most of the IncRNAs are not co-expressed with neighbouring proteincoding genes.

Conclusions: Alongside substantially expanding the ruminant IncRNA repertoire, the outcomes of our analysis demonstrate that stochastic sampling can be partly overcome by combining RNA-seq datasets from related species. This has practical implications for the future discovery of IncRNAs in other species.

\section{Background}

Mammalian transcriptomes include many long non-coding RNAs (lncRNAs), a collective term for transcripts of more than 200 nucleotides that resemble mRNAs (many being $3^{\prime}$ polyadenylated, $5^{\prime}$ capped and spliced) but do not encode a protein product [1]. Proposed functional roles of IncRNAs include transcriptional regulation,

\footnotetext{
*Correspondence: stephen.bush@ndm.ox.ac.uk; stephen.bush@roslin. ed.ac.uk; david.hume@uq.edu.au; david.hume@roslin.ed.ac.uk ${ }^{\dagger}$ Emily L. Clark and David A. Hume have contributed equally to this work

${ }^{1}$ The Roslin Institute, University of Edinburgh, Easter Bush Campus, Edinburgh, Midlothian EH25 9RG, UK

Full list of author information is available at the end of the article
}

epigenetic regulation, intracellular trafficking and chromatin remodelling (see reviews [2-9]). Some view lncRNAs as transcriptional noise [10, 11]. Full length lncRNAs are difficult to assemble: many are expressed at low levels [12], with high tissue-specificity [13, 14], at specific developmental time points (e.g. [15-17]), and with few signs of selective constraint [18, 19]. Many are also expressed transiently, and thus may be partly degraded by the exosome complex [20].

The initial recognition of lncRNAs as widespread and bona fide outputs of mammalian transcription was based on the isolation and sequencing of large numbers of mouse and human full-length cDNAs [21-23], many of 
which were experimentally validated [24] and shown to participate in sense-antisense pairs [25]. They were captured in significant numbers because the cDNA libraries were subtracted to remove abundant transcripts. More recent studies have used RNA-sequencing (RNA-seq) to assemble larger catalogues of lncRNAs [26]. Because of the power-law relationship of individual transcript abundance in mammalian transcriptomes [27], unless sequencing is carried out at massive depth, the exons of lowly-abundant transcripts (such as lncRNAs) are subject to stochastic sampling and are detected inconsistently between technical replicates of the same sample [28]. RNA-seq is also a relatively inaccurate means of reconstructing the $5^{\prime}$ ends of transcripts [29]. To overcome this constraint, the FANTOM Consortium supplemented RNA-seq with Cap Analysis of Gene Expression (CAGE) data, characterising-in humans-a $5^{\prime}$-complete lncRNA transcriptome [30].

RNA-seq libraries from multiple tissues, cell types and developmental stages are commonly pooled to maximise the number of lncRNA gene models assembled. Genomewide surveys have expanded the lncRNA repertoire of livestock species such as cattle (18 tissues, sequenced at approximately 40 to 100 million reads each) [31], pig (10 tissues, sequenced at approximately 6 to 40 million reads each) [32], horse (8 tissues, sequenced at approximately 20 to 200 million reads each) [33] and sheep (8 tissues, sequenced at approximately 16 million reads each) [34], complementing tissue-specific lncRNA catalogues of, for example, cattle muscle [35, 36] and skin [37], and pig adipose [38, 39], liver [40] and testis [41].

The low level of lncRNA conservation (at some loci, it appears that only the act of transcription, rather than the transcript sequence itself, is functionally relevant [42]) reduces the utility of comparative analysis of the large RNA-seq datasets available from humans [30, 43] and mouse [44]. Among 200 human and mouse lncRNAs that are each characteristic of specific immune cell types, there was less than $1 \%$ sequence conservation [45].

Here we focus on more closely related species. We have generated atlases of gene expression for the domestic sheep, Ovis aries [46], and the goat, Capra hircus (manuscript in preparation). Since these two species are closely related (sharing a common ancestor less than 10 million years ago (mya) [47]) and their respective RNA-seq datasets contain many of the same tissues, it is possible to use data from one species to infer the presence of lncRNAs in the other. Cattle and humans are more distantly related to small ruminants, nevertheless they are substantially more similar than mice. We extend our approach by using existing human and cattle lncRNA datasets to identify a consensus ruminant lncRNA transcriptome, and use the sheep transcriptional atlas to confirm that candidate lncRNAs identified by cross-species inference are reproducibly expressed. The lncRNA catalogues that we have generated in the sheep and goat are of interest in themselves [48] and contribute valuable information to the Functional Annotation of Animal Genomes (FAANG) project $[49,50]$.

\section{Methods}

\section{Sheep RNA-sequencing data}

Previously, we created an expression atlas for the domestic sheep [46], using RNA-seq data that were largely collected from adult Texel $\times$ Scottish Blackface (TxBF) sheep. Experimental protocols for tissue collection, cell isolation, RNA extraction, library preparation, RNA sequencing and quality control are as previously described [46], and independently available on the FAANG Consortium website (http://www.ftp.faang. ebi.ac.uk/ftp/protocols). All RNA-seq libraries were prepared by Edinburgh Genomics (Edinburgh Genomics, Edinburgh, UK) and sequenced using the Illumina HiSeq 2500 sequencing platform (Illumina, San Diego, USA). The majority of these libraries were sequenced to a depth of more than 25 million paired-end reads per sample using the Illumina TruSeq mRNA library preparation protocol (polyA-selected) (Illumina; Part: 15031047, Revision E). A subset of 11 transcriptionally rich 'core' tissues (bicep muscle, hippocampus, ileum, kidney medulla, left ventricle, liver, ovary, reticulum, spleen, testes, thymus), plus one cell type under two conditions (bone marrow derived macrophages (BMDM), unstimulated and $7 \mathrm{~h}$ after simulation with lipopolysaccharide (LPS)), were sequenced to a depth of more than 100 million paired-end reads per sample using the Illumina TruSeq total RNA library preparation protocol (rRNA-depleted) (Illumina; Part: 15031048, Revision E). The choice of 'core' tissues was informed by those included in previous human and mouse transcriptional atlases [51, 52], and reflect the high proportion of protein-coding genes transcribed in each species. Other samples-in particular, bone marrow derived macrophages-were included in the sheep expression atlas as a known source of novel mRNAs [53]. For characterising lncRNAs, we assume that the transcriptional diversity of protein-coding RNAs reflects the transcriptional diversity of non-coding RNAs.

Sample metadata for all tissue and cell samples are deposited in the EBI BioSamples database under submission identifier GSB-718 (https://www.ebi.ac.uk/biosamples/groups/SAMEG317052). The raw read data, as.fastq files, are deposited in the European Nucleotide Archive (ENA) under study accession PRJEB19199 (http://www. ebi.ac.uk/ena/data/view/PRJEB19199). 


\section{Goat RNA-sequencing data}

All RNA-seq libraries for goat were prepared by Edinburgh Genomics (Edinburgh Genomics, Edinburgh, UK) (as above) and sequenced using the Illumina HiSeq 4000 sequencing platform (Illumina, San Diego, USA). These libraries were sequenced to a depth of more than 30 million paired-end reads per sample using the Illumina TruSeq mRNA library preparation protocol (polyAselected) (Illumina; Part: 15031047, Revision E). Sample metadata for all tissue and cell samples are deposited in the EBI BioSamples database under submission identifier GSB-2131 (https://www.ebi.ac.uk/biosamples/groups/ SAMEG330351). The raw read data, as.fastq files, are deposited in the ENA under study accession PRJEB23196 (http://www.ebi.ac.uk/ena/data/view/PRJEB23196).

\section{Identifying candidate IncRNAs in sheep and goats}

Previously, we described an RNA-seq processing pipeline for sheep [46] - using the HISAT2 aligner [54] and StringTie assembler [55] - for generating a uniform, nonredundant set of de novo assembled transcripts. The same pipeline was applied to the goat RNA-seq data. This produced a single file per species, merged.gtf; that is, the output of StringTie-merge, which collates every transcript model from the 54 goat assemblies (each assembly being both individual- and tissue-specific), and 429 of the 441 assemblies within the sheep expression atlas [46] (12 sheep libraries were not used for this purpose since they were replicates of pre-existing bone marrow-derived macrophage libraries, which were prepared by using an mRNA-seq rather than a total RNA-seq protocol). Not all transcript models in either GTF will be stranded. This is because HISAT2 infers the transcription strand of a given transcript by reference to its splice sites; this is not possible for single exon transcripts, which are un-spliced.

The GTF was parsed to distinguish candidate lncRNAs from assembly artefacts, and from other RNA, by applying the filter criteria of Ilott et al. [56], excluding gene models that (a) were longer than $200 \mathrm{bp}$, (b) overlapped (by more than $1 \mathrm{bp}$ on the same strand) any coordinates annotated as 'protein-coding' or 'pseudogene' (these classifications are explicitly stated in the Ensembl-hosted Oar v3.1 annotation and assumed true of all gene models in the ARS1 annotation), or (c) were associated with multiple transcript models (which are more likely to be spurious). For single-exon gene models, we used a more conservative length threshold of $500 \mathrm{bp}$-the lower threshold of $200 \mathrm{bp}$ could be otherwise met by a single pair of reads. We further excluded any novel gene model that was previously considered protein-coding in each species' expression atlas (as described in [46]); these models contain an ORF encoding a peptide homologous to a ruminant protein in the
NCBI nr database [46]. These criteria establish longlists of 30,677 candidate sheep lncRNAs $(14,862$ of which are multi-exonic) and 7671 candidate goat lncRNAs (3289 of which are multi-exonic). The sheep genome, Oar v3.1, already contains 1858 lncRNA models, of which the StringTie assembly precisely reconstructs $1402(75 \%)$. In spite of this pre-existing support, these models were included on the sheep longlist for independent verification. The goat genome, by contrast, was annotated with a focus on protein-coding gene models [57], by consolidating protein and cDNA alignments-from exonerate [58] and tblastn [59] - with the annotation tool EVidence Modeller (EVM) [60]. Consequently, there are no unambiguous lncRNAs in the associated GTF (http://www. ftp.ncbi.nlm.nih.gov/genomes/all/GCF/001/704/415/ GCF_001704415.1_ARS1/GCF_001704415.1_ARS1_ genomic.gff.gz, accessed 23rd October 2017) (unlike the Ensembl-hosted sheep annotation, the goat annotation is currently only available via NCBI).

Each longlist of candidates was assessed for coding potential using three different tools: CPAT v1.2.3 [61], which assigns coding probabilities to a given sequence based on differential hexamer usage [62] and Fickett TESTCODE score [63], PLEK v1.2, a support vector machine classifier using k-mer frequencies [64], and CPC v0.9-r2 [65], which was used in conjunction with the non-redundant sequence database, UniRef90 (the Uniref Reference Cluster, a clustered set of sequences from the UniProt KnowledgeBase that constitutes a comprehensive coverage of sequence space at a resolution of $90 \%$ identity) [66, 67] (http://www.ftp.uniprot.org/ pub/databases/uniprot/uniref/uniref90/uniref90.fasta. gz, accessed 18th August 2017). CPC scores putatively coding sequences positively and non-coding sequences negatively. We retained only those sequences with a CPC score less than -0.5 (consistent with previous studies $[31,37]$ ) and a CPAT probability higher than 0.58 (after creating sheep-specific coding and non-coding CPAT training data, from Oar v3.1 CDS and ncRNA, this cutoff is the intersection of two receiver operating characteristic curves, obtained using the R package ROCR [68]; this cut-off is also used for the goat data, as there are insufficient non-coding training data for this species).

For each remaining gene model, we concatenated its exon sequence and identified the longest ORF within it. Should CPC, CPAT or PLEK make a false positive classification of 'non-coding', this translated ORF was considered the most likely peptide encoded by the gene. Gene models were further excluded if the translated ORF (a) contained a protein domain, based on a search by HMMER v3.1b2 [69] of the Pfam database of protein families, v31.0 [70], with a threshold E-value of $1 \times 10^{-5}$, or (b) shared homology with a known peptide 
in the Swiss-Prot March 2016 release [71, 72], based on a search with BLAST + v2.3.0 [59]: blastp with a threshold E-value of $1 \times 10^{-5}$. Shortlists of 12,296 (sheep) and 2657 (goat) candidate lncRNAs-each with three independent 'non-coding' classifications and no detectable blastp and HMMER hits-are in Tables S1 and S2 [see Additional file 1: Table S1 and Additional file 2: Table S2], respectively.

\section{Classification of IncRNAs}

Using the set of Oar v3.1 transcription start sites (TSS), which was obtained from Ensembl BioMart [73], and the set of ARS1 gene start sites (http://www.ftp.ncbi.nlm.nih. gov/genomes/all/GCF/001/704/415/GCF_001704415.1_ ARS1/GCF_001704415.1_ARS1_genomic.gff.gz, accessed 23rd October 2017), we classified novel candidate lncRNAs for each species as done in [74], as either (a) sense or antisense (if the coordinates of the lncRNAs overlap, or are encapsulated by, a known gene on the same, or opposite, strand), (b) up- or downstream, and on the same or opposite strand (if $<5 \mathrm{~kb}$ from the nearest TSS), or (c) intergenic (if $\geq 5,10,20,50,100,500 \mathrm{~kb}$ or $1 \mathrm{Mb}$ from the nearest TSS, irrespective of strand). The HISAT2/StringTie pipeline, used to generate these transcript models, could not infer the transcription strand in all cases, particularly for single-exon transcripts. Accordingly, some lncRNAs will overlap the coordinates of a known gene, but its strandedness with respect to that gene-whether it is sense or antisense-will be unknown.

\section{Conservation of IncRNAs in terms of sequence}

To assess the sequence-level conservation of sheep and goat lncRNA transcripts, we obtained human lncRNA sequences from two databases, NONCODE v5 [75] (http://www.noncode.org/datadownload/NONCODEv5_human.fa.gz, accessed 27th September 2017) and lncRNAdb v2.0 [76] (http:// www.Incrnadb.com/media/cms_page_media/10651/ Sequences_lncrnadb_27Jan2015.csv, accessed 27th September 2017) (which contain 172,216 and 152 lncRNAs, respectively). A previous study of lncRNAs in cattle [31] also generated a conservative set of 9778 lncRNAs, all of which were detectably expressed in at least one of the 18 tissues (read count $>25$ in each of three replicates per tissue). These sets of sequences constitute three independent BLAST databases. For each sheep and goat lncRNA, blastn searches [59] were made against each database using an arbitrarily high E-value of 10 , as substantial sequence-level conservation was not expected.

\section{Conservation of IncRNAs in terms of synteny}

For each of the human (GRCh38.p10), sheep (Oar v3.1), cattle (UMD3.1) and goat (ARS1) reference genomes, we established those regions in each pairwise comparison where gene order is conserved, obtaining reference annotations from Ensembl BioMart v90 [73] (sheep, cattle and human) and NCBI (goat; http://www.ftp.ncbi.nlm.nih. gov/genomes/all/GCF/001/704/415/GCF_001704415.1_ ARS1/GCF_001704415.1_ARS1_genomic.gff.gz, accessed 27th September 2017). By advancing a sliding window across each chromosome gene-by-gene from the $5^{\prime}$ end, we identified the first upstream and first downstream gene of each focal gene, irrespective of strand. For the purpose of this analysis, the first and last genes on each chromosome are excluded, since they have no upstream or downstream neighbour, respectively. Then, for each pairwise species comparison, we determined which sets of blocks were present in both-that is, where the HGNC symbols for upstream gene/focal gene/downstream gene were identical. These syntenic blocks, of three consecutive genes each, are regions in the genome where gene order is conserved both up- and downstream of a focal gene: between sheep and cattle, there are 2927 regions (comprising 5601 unique genes); sheep and goat, 2038 regions (3883 unique genes); cattle and goat, 2982 regions (5258 unique genes); sheep and human, 380 regions (930 unique genes); goat and human, 527 regions (1262 unique genes); cattle and human, 443 regions (1063 unique genes). If in each syntenic block a lncRNA was found between the upstream and focal gene, or the focal and downstream gene, in only one of the two species, a global alignment was made between the transcript and the intergenic region of the corresponding species. Alignments were made using the Needleman-Wunsch algorithm, as implemented by the 'needle' module of EMBOSS v6.6.0 [77], with default parameters. By effectively treating lncRNA transcripts as if they were CAGE tags (that is, short reads of 20 to 50 nucleotides [78]), we considered successful alignments as those containing one or more consecutive runs of 20 identical residues, without gaps. The probability that a transcript randomly matches 20 consecutive residues, within a pre-defined region, is extremely low.

For successful alignments, the target sequence (that is, an extract from the intergenic region) was considered to be a novel lncRNA. For this analysis, the sheep and goat lncRNAs used are those from their respective shortlists [see Additional file 1: Table S1 and Additional file 2: Table S2]. Locations of IncRNAs in other species are obtained from previous studies that applied similarly conservative classification criteria. For cattle, 9778 lncRNAs were obtained [31], each of which were longer than $200 \mathrm{bp}$, considered non-coding by the classification tools CPC [65] and CNCI [79], lacked sequence similarity to the NCBI nr [46] and Pfam databases [70], and had a normalised read count higher than 25 in at least two of three 
replicates per tissue for 18 tissues. For human, 17,134 lncRNAs were obtained [80], each of which were assembled from transfrags longer than $250 \mathrm{bp}$, considered non-coding by the classification tool CPAT [61], lacked sequence similarity to the Pfam database [70], and had active transcription confirmed by intersecting intervals surrounding the transcriptional start site with chromatin immunoprecipitation and sequencing (ChIP-seq) data from 13 cell lines.

\section{Quantification of expression level}

For the 11 'core' tissues of the sheep expression atlas, plus unstimulated and LPS-stimulated BMDMs (for details Table S2 in [46] and available under ENA accession PRJEB19199), expression was quantified using Kallisto v0.43.0 [81] with a $k$-mer index $(k=31)$ derived after supplementing the Oar v3.1 reference transcriptome with the shortlist of 11,646 novel sheep lncRNA models (Additional file 1: Table S1) and those lncRNAs assembled in either human $(n=18)$, goat $(n=164)$, or cattle $(\mathrm{n}=1219)$, and which map to a conserved region of the sheep genome [see Additional file 3: Table S3]. Oar v3.1 transcripts were obtained from Ensembl v90 [73] in the form of separate files for 22,823 CDS (http://www.ftp. ensembl.org/pub/release-90/fasta/ovis_aries/cds/Ovis_ aries.Oar_v3.1.cds.all.fa.gz, accessed 27th September 2017) and 6005 ncRNAs (http://www.ftp.ensembl.org/ pub/release-90/fasta/ovis_aries/ncrna/Ovis_aries.Oar v3.1.ncrna.fa.gz, accessed 27th September 2017).

An equivalent set of expression estimates was made for goat, across the 21 tissues and cell types of the goat expression atlas (i.e., 54 RNA-seq libraries available under ENA accession PRJEB23196). 47,193 transcripts, from assembly ARS1, were obtained from NCBI (http://www. ftp.ncbi.nlm.nih.gov/genomes/all/GCF/001/704/415/ GCF_001704415.1_ARS1/GCF_001704415.1_ARS1_ rna.fna.gz, accessed 27th September 2017), and supplemented both with the shortlist of 2657 novel goat lncRNA models [see Additional file 2: Table S2], and those lncRNAs assembled in human $(\mathrm{n}=15)$, sheep $(n=507)$, or cattle $(n=1213)$ [see Additional file 3: Table S3]. After quantification in each species, transcript-level abundances were summarised to the gene-level.

\section{Categorisation of expression profiles}

Expression levels were categorised as done in the Human Protein Atlas [82], and as previously employed in the Sheep Gene Expression Atlas [46]. Each gene is considered to have either no detectable expression (average $\mathrm{TPM}<1$, a threshold chosen to minimise the influence of stochastic sampling), low expression (10>average $\mathrm{TPM} \geq 1)$, medium expression $(50>$ average $\mathrm{TPM}>10)$, or high expression (average $\mathrm{TPM} \geq 50$ ). Three sample specificity indices were calculated for each gene, as in [46]. These include $t a u$, a scalar measure of expression breadth bound between 0 (for housekeeping genes) and 1 (for genes expressed in one sample only) [83], and the mean TPM (across all samples) divided by the median TPM (across all tissues). Genes with greater sample specificity will have a more strongly skewed distribution (i.e. a higher mean and a lower median), and so the larger the ratio, the more sample-specific the expression. To avoid undefined values, if median TPM is equal to 0 , it is set to 0.01 .

We also calculated a preferential expression measure (PEM), as in [84]. PEM is calculated per tissue per gene (unlike tau) and quantifies the expression of a given gene in a given tissue in relation to its average expression across all tissues (more tissue-specific genes will have higher PEM values for that tissue). For each gene $i$ in tissue $t_{i}, \operatorname{PEM}\left(t_{i}\right)=\mathrm{S}-\mathrm{A}$, where $\mathrm{S}=$ expression of gene $i$ in tissue $t_{i}$, and $\mathrm{A}=$ arithmetic mean expression of gene $i$ across all tissues. As there are biological replicates of each tissue, we considered $S$ to be the mean TPM per gene and $A$ to be the mean of all values of $S$. Before PEM was calculated, all values less than 1 were considered to be 1 , and a $\log _{2}$-transformation was then applied.

Each gene is also assigned one or more categories, to allow an at-a-glance overview of its expression profile: (a) 'tissue enriched' (expression in one tissue at least five-fold higher than all other tissues ['tissue specific' if all other tissues have 0 TPM]), (b) 'tissue enhanced' (five-fold higher average TPM in one or more tissues compared to the mean TPM of all tissues with detectable expression [this category is mutually exclusive with 'tissue enriched'), (c) 'group enriched' (five-fold higher average TPM in a group of two or more tissues compared to all other tissues ('groups' are analogous to organ systems, and are as described in the sheep expression atlas [46]), (d) mixed expression (detected in one or more tissues and neither of the previous categories), (e) 'expressed in all' (more than 1 TPM in all tissues), and (f) 'not detected' (less than 1 TPM in all tissues).

\section{Network analysis}

Network analysis of the sheep expression level data was performed using Graphia Professional (Kajeka Ltd, Edinburgh, UK), a commercial version of BioLayout Express ${ }^{3 \mathrm{D}}$ $[85,86]$. A correlation matrix was built for each geneto-gene comparison, which was then filtered by removing all correlations below a given threshold (Pearson's $r<0.95$ ). A network graph was constructed by connecting nodes (genes) with edges (correlations above the threshold). The local structure of the graph-that is, clusters of co-expressed genes-was interpreted by applying the 
Markov clustering (MCL) algorithm [87] at an inflation value (which determines cluster granularity) of 2.2 .

\section{Enrichment of IncRNAs in the vicinity of protein-coding genes}

To test whether lncRNAs that are co-expressed with protein-coding genes are more likely to be closer to them (from which we can infer that they are more likely to have been derived from an enhancer sequence affecting that protein-coding gene), we used a randomisation test as in [88]. First, we obtained clusters of co-expressed genes from a network graph of the sheep expression level dataset (see above). We then calculated $q$, the number of times the distance between each lncRNA and the nearest protein-coding gene within the same cluster was higher than the distance between each lncRNA and the nearest gene within $s=1000$ randomly selected, equally sized, subsets of protein-coding genes, drawn from the same chromosome as each lncRNA. Letting $r=s-q$, then the $\mathrm{p}$-value of this test is $r+1 / s+1$.

\section{Results and discussion}

Identifying IncRNAs in the sheep and goat transcriptomes

Previously, we created an expression atlas for the domestic sheep [46], using both polyadenylated and rRNAdepleted RNA-seq data that were collected primarily from three male and three female adult Texel $\times$ Scottish Blackface $(\mathrm{T} \times \mathrm{BF})$ sheep at 2 years of age: 441 RNA-seq libraries in total, comprising five cell types and multiple tissues spanning all major organ systems and several developmental stages, from embryonic to adult. To complement this dataset, we also created a smaller-scale expression atlas-of 54 mRNA-seq libraries-from 6-day old crossbred goats, which will be the subject of a dedicated analysis. For both species, each RNA-seq library was aligned against its reference genome (Oar v3.1 and ARS1, for sheep and goat, respectively) using HISAT2 [54], with transcripts assembled using StringTie [55]. This pipeline produced a non-redundant set of de novo gene and transcript models, as previously described [46], and expanded the set of transcripts in each reference genome to include $a b$ initio lncRNA predictions and novel protein-coding genes. As the primary purpose of the sheep expression atlas was to improve the functional characterisation of the protein-coding transcriptome, the novel sheep protein-coding transcript models generated by this pipeline are discussed in [46] (novel protein-coding transcripts for goats will be discussed in a dedicated analysis of the protein-coding goat transcriptome).

Using similar filter criteria to a previous study [56], the de novo gene models were parsed to create longlists of
30,677 (sheep) and 7671 (goat) candidate lncRNAs, each of which was longer than $200 \mathrm{bp}$ and was not associated, on the same strand, with a known protein-coding locus. The fourfold difference in the length of each longlist can be attributed to the relative size of each dataset. The sheep atlas contains 8 times as many RNA-seq libraries, spans multiple developmental stages (from embryonic to adult), and has a subset of its samples that was specifically prepared to ensure the comprehensive capture of ncRNAs - unlike any sample in the goat dataset, this subset is sequenced at a fourfold higher depth ( $>100$ million reads, rather than $>25$ million reads) using a total RNAseq, rather than mRNA-seq, protocol.

Each model on both longlists was assessed for coding potential using the classification tools CPC [65], CPAT [61] and PLEK [64], alongside homology searches of its longest ORF-with blastp [59] and HMMER [69]-to known protein and domain sequences (within the SwissProt [71, 72] and Pfam-A [70] databases, respectively). Those gene models classified as non-coding by CPC, CPAT and PLEK, and having no detectable blastp and HMMER hits, are considered novel lncRNAs.

This pipeline creates shortlists of 12,296 (sheep) and 2657 (goat) lncRNAs [see Additional file 1: Table S1 and Additional file 2: Table S2], respectively), representing approximately $40 \%$ (sheep) and 35\% (goat) of the gene models on each longlist. The mean gene length is similar in both shortlists-6.7 kb (sheep) and $8.8 \mathrm{~kb}$ (goat)-as its summed exon length, averaging $1.2 \mathrm{~kb}$ in each species.

Consistent with previous analyses in several other species [31, 89], 6956 (57\%) of the sheep lncRNAs, and 1284 (48\%) of the goat lncRNAs, were single-exonic. For sheep, the shortlist contains 11,646 previously unknown lncRNA models and provides additional evidence for 650 existing Oar v3.1 lncRNA models (Additional file 1: Table S1). A small proportion of longlisted gene models were considered non-coding by at least one of CPC, CPAT or PLEK, nevertheless they showed some degree of sequence homology to either a known protein or protein domain: for sheep, 226 (including 13 existing Oar v3.1 models) [see Additional file 1: Table S4], and for goats, 153 [see Additional file 2: Table S5]. The number of novel lncRNAs identified is also given per chromosome, for sheep [see Additional file 1: Table S6] and for goat [see Additional file 2: Table S7] and per type, for sheep [see Additional file 1: Table S8] and for goat [see Additional file 2: Table S9], the majority of which-in both speciesare found in intergenic regions, 10 to $100 \mathrm{~kb}$ from the nearest gene. Overall, the addition of these lncRNA models increases the total number of genes in the reference annotation by approximately $30 \%$ (sheep) and $12 \%$ (goat). 


\section{The sets of ab initio sheep and goat IncRNAs only minimally overlap at the sequence level}

Even with full length cDNA sequences, comparative analysis revealed that the majority are not conserved between species: estimates of the proportion of human lncRNAs with mouse counterparts range from 14 [90]-27\% [23] (see also review [19]). When comparing the sets of sheep and goat lncRNAs, few predicted transcripts-in either species-show sequence-level similarity either to each other or to other closely or distantly related species (cattle and humans, respectively, which shared a common ancestor with sheep and goats approx. 25 and 95 mya [47]). Of the 12,296 shortlisted sheep lncRNAs, less than half $(\mathrm{n}=5139$, i.e. $42 \%)$ had any detectable pairwise alignment-of any quality and of any length-to either the shortlisted goat lncRNAs, a set of 9778 cattle lncRNAs from a previous study [31] or two sets of human lncRNAs (Fig. 1 and Table S10 [see Additional file 1: Table S10]).

High confidence is possible only for a small proportion of these alignments, i.e. the alignment has a \% identity higher than 50\% within an alignment longer than 50\% of the length of the target sequence. Of the 5139 sheep lncRNAs that could be aligned to any species, only 293 $(5.7 \%)$ could be aligned with high confidence to goat and $265(5.2 \%)$ to cattle transcripts. Similarly, of the sheep lncRNAs that could be aligned to either of two human
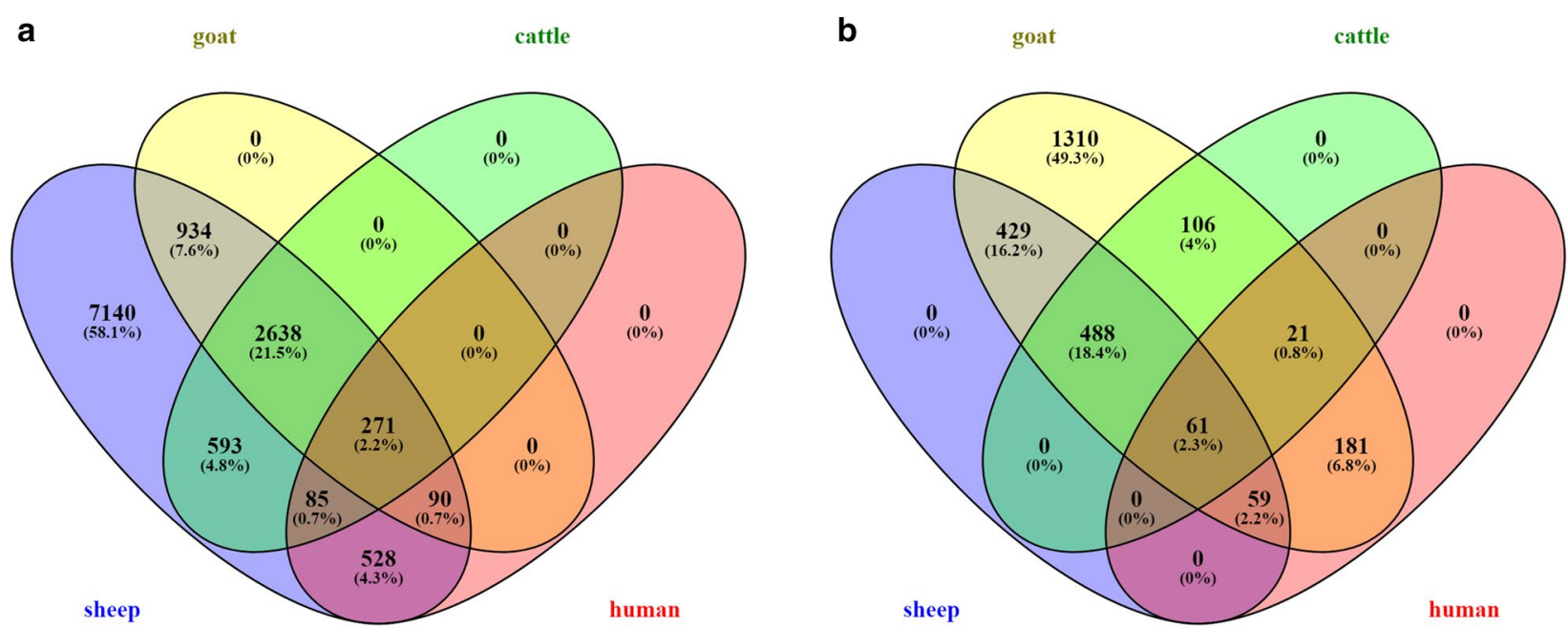

C

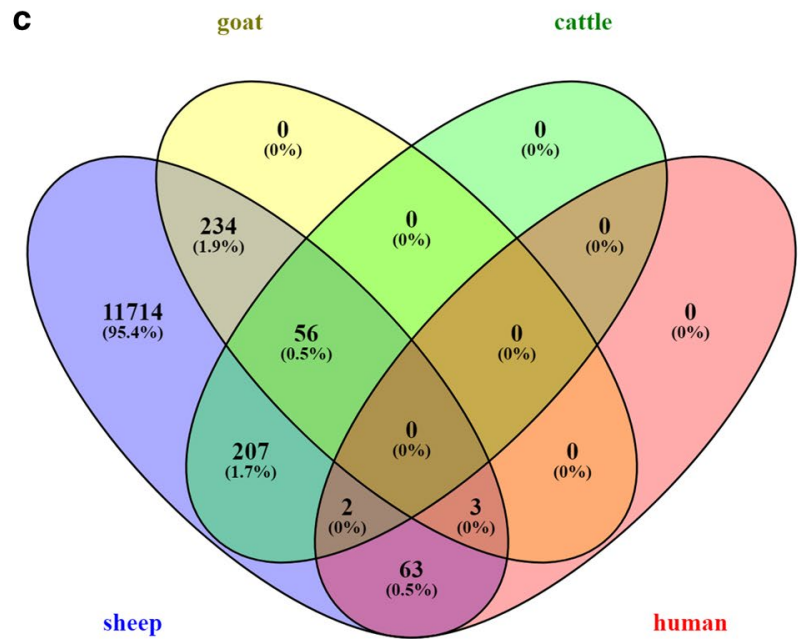

d
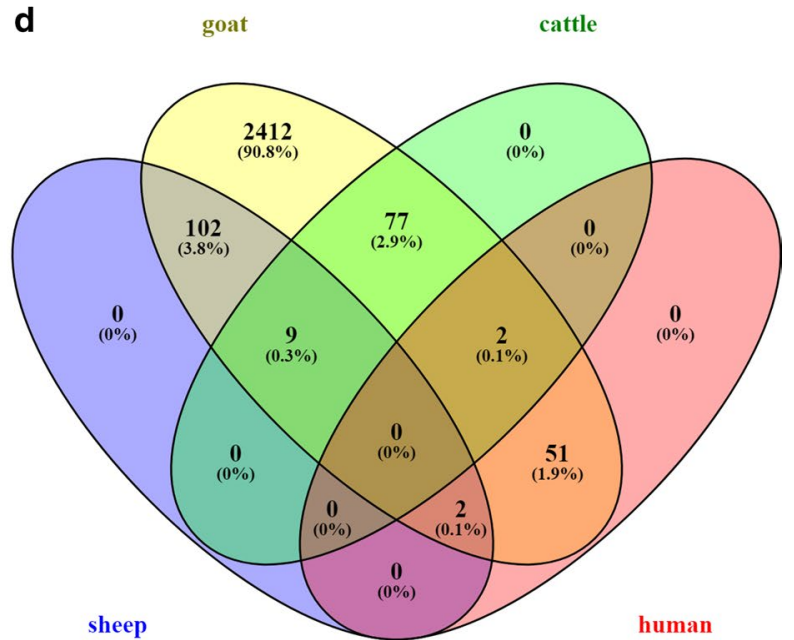

Fig. 1 Minimal overlap of IncRNAs at the sequence level. Venn diagrams show the number of sheep (a and $\mathbf{c}$ ) or goat (b and $\mathbf{d})$ IncRNAs that can be aligned - either with an alignment of any length or quality (A and B), or with $\geq 50 \%$ identity over $\geq 50 \%$ of the length of the target sequence (c and $\mathbf{d}$ ) - to either shortlist of goat (a and $\mathbf{c}$ ) or sheep (b and $\mathbf{d}$ ) IncRNAs, and to sets of cattle and human IncRNAs from previous studies. The majority (58\% of sheep IncRNAs, and 49\% of goat IncRNAs) have no associated alignment. Alignments are detailed in Additional file 1:Table S10 (sheep) and Additional file 1: Table S11 (goat) 
lncRNA databases-NONCODE [75] and lncRNAdb [76]-68 (1.6\% of the total alignable lncRNA) aligned with high confidence to the NONCODE database, and none to the lncRNAdb. Similar findings are observed with the 2657 shortlisted goat lncRNAs: 1343 (50.5\%) had a detectable pairwise alignment, of any quality, to either set of sheep, cattle or human lncRNAs. However, of these 1343 lncRNAs, only 113 (8.4\%) aligned with high confidence to sheep, $88(6.6 \%)$ to cattle, $55(4.1 \%)$ to the human NONCODE database, and $1(0.1 \%)$ to the human lncRNAdb database (Fig. 1 and Table S11 [see Additional file 2: Table S11]). These observations allow for two possibilities. First, lncRNAs may, in general, be poorly conserved at the sequence level, which is consistent with previous findings $[18,19]$ and the observation that only $6 \%$ of the sheep/goat alignments have more than $50 \%$ reciprocal identity. However, an alternative is that in spite of the apparent depth of coverage, we have only assembled a subset of the total lncRNA transcriptome in each species.

IncRNAs not captured by the RNA-seq libraries of one species can be found using data from a related species

A reasonable a priori prediction is that lncRNAs conserved in a closely related species-which are more likely to be functionally relevant-are also more similarly expressed. Whereas human and mouse lncRNAs identified as full length cDNAs were generally less conserved between species than the $5^{\prime}$ and $3^{\prime} \mathrm{UTR}$ of protein-coding transcripts, their promoters were more highly conserved than those of protein-coding transcripts, some extending as far as chicken [44, 91]. These findings suggested that the large majority of lncRNAs that were analyzed displayed positional conservation across species. Accordingly, rather than comparing the similarity of two sets of lncRNA transcripts, we mapped the lncRNAs assembled in one species (e.g. sheep) to the genome of another (e.g. goat), deriving confidence in the mapping location from synteny.

For each of the pairwise sheep/cattle, sheep/goat, cattle/goat, sheep/human, goat/human, and cattle/human comparisons, we identified sets of syntenic blocks: regions in the genome where gene order is conserved both up- and downstream of a focal gene (see Table 1 and the "Methods" section).

In the sheep/cattle comparison, approximately $5 \%$ of the syntenic blocks contain at least one lncRNA with a relative position conserved in both species, either upstream $(n=139 \operatorname{lncRNAs})$ or downstream $(n=141)$ of the central gene in each block [see Additional file 3: Table S12]. In the sheep/goat and cattle/goat comparisons, respectively, approximately 2 and $3 \%$ of the syntenic blocks contain a lncRNA (for sheep/goat, $n=42$ upstream, 40 downstream; for cattle/goat, 86 upstream, 83 downstream) [see Additional file 3: Tables S13 and S14]. With increased species divergence, far fewer lncRNAs have relative positions conserved in either the upstream or downstream positions of the sheep/human, goat/human and cattle/human syntenic blocks (typically, $<1 \%$ of the syntenic blocks contain a lncRNA) [see Additional file 3: Tables S15, S16 and S17]. These comparatively small proportions highlight the minimal overlap between each set of assembled transcripts, which is consistent with stochastic assembly-lncRNAs expected to be present in a particular location are captured in only one species, not both. As such, very few lncRNAs in either of the sheep, goat and cattle subsets have evidence of both shared sequence homology and conserved synteny. When comparing sheep and cattle, 16 unique lncRNAs have high-confidence pairwise alignments within a region of conserved synteny, and six when comparing sheep and goat [see Additional file 3: Table S18].

In most of the syntenic blocks examined, if a lncRNA was detected in one location in one species (either up- or

Table 1 Comparatively few IncRNAs appear positionally conserved, suggesting minimal overlap between each species' set of transcripts

\begin{tabular}{llllll}
\hline Species 1 & Species 2 & $\begin{array}{l}\text { Number of syntenic blocks } \\
\text { (i.e. three conserved con- } \\
\text { secutive genes) }\end{array}$ & $\begin{array}{l}\text { Number of unique pro- } \\
\text { tein-coding genes in the } \\
\text { set of syntenic blocks }\end{array}$ & $\begin{array}{l}\text { Total number of positionally } \\
\text { conserved IncRNAs in the set } \\
\text { of syntenic blocks (in either the } \\
\text { up- or downstream position) }\end{array}$ & $\begin{array}{l}\text { \% of syntenic blocks } \\
\text { with at least one } \\
\text { positionally conserved } \\
\text { IncRNA }\end{array}$ \\
\hline Sheep & Cattle & 2927 & 5601 & 280 & 9.57 \\
Sheep & Goat & 2038 & 3883 & 82 & 4.02 \\
Sheep & Human & 380 & 930 & 8 & 2.11 \\
Goat & Cattle & 2982 & 5258 & 169 & 5.67 \\
Goat & Human & 527 & 1262 & 2 & 0.38 \\
Cattle & Human & 443 & 1063 & 5 & 1.13 \\
\hline
\end{tabular}

The results suggest that the IncRNAs that are expected to be found at a given genomic location are captured in only one species, not both, consistent with the stochastic sampling of IncRNAs by RNA-seq libraries 
Table 2 Direct mapping of IncRNA transcripts to the genome of another species

\begin{tabular}{|c|c|c|c|c|c|c|}
\hline $\begin{array}{l}\text { Species } 1 \text { (in } \\
\text { which IncRNAs } \\
\text { are captured } \\
\text { by RNA-seq } \\
\text { libraries) }\end{array}$ & $\begin{array}{l}\text { Species } 2 \text { (in } \\
\text { which IncRNA } \\
\text { can be inferred) }\end{array}$ & $\begin{array}{l}\text { Number of IncRNA models } \\
\text { detected within a region } \\
\text { of conserved synteny } \\
\text { between species } 1 \text { and } 2 \text {, } \\
\text { but not captured by the } \\
\text { RNA-seq libraries of spe- } \\
\text { cies } 2\end{array}$ & $\begin{array}{l}\text { Number } \\
\text { of IncRNA } \\
\text { models } \\
\text { from species } \\
1 \text { mapped } \\
\text { to the genome } \\
\text { of species } 2\end{array}$ & $\begin{array}{l}\% \text { of IncRNA } \\
\text { models } \\
\text { detected } \\
\text { by direct } \\
\text { genome map- } \\
\text { ping }\end{array}$ & $\begin{array}{l}\text { Number of inter- } \\
\text { genic regions } \\
\text { in the syntenic } \\
\text { blocks conserved } \\
\text { between these two } \\
\text { species }\end{array}$ & $\begin{array}{l}\% \text { of intergenic } \\
\text { regions in which a } \\
\text { IncRNA from spe- } \\
\text { cies } 1 \text { is inferred } \\
\text { in species } 2\end{array}$ \\
\hline \multirow[t]{3}{*}{ Cattle } & Goat & 2593 & 1213 & 46.78 & 5964 & 20.34 \\
\hline & Human & 163 & 20 & 12.27 & 886 & 2.26 \\
\hline & Sheep & 2939 & 1219 & 41.48 & 5854 & 20.82 \\
\hline \multirow[t]{3}{*}{ Goat } & Cattle & 2593 & 286 & 11.03 & 5964 & 4.8 \\
\hline & Human & 76 & 9 & 11.84 & 1054 & 0.85 \\
\hline & Sheep & 991 & 164 & 16.55 & 4076 & 4.02 \\
\hline \multirow[t]{3}{*}{ Human } & Cattle & 163 & 16 & 9.82 & 886 & 1.81 \\
\hline & Goat & 76 & 15 & 19.74 & 1054 & 1.42 \\
\hline & Sheep & 93 & 18 & 19.35 & 760 & 2.37 \\
\hline \multirow[t]{3}{*}{ Sheep } & Cattle & 2939 & 775 & 26.37 & 5854 & 13.24 \\
\hline & Goat & 991 & 507 & 51.16 & 4076 & 12.44 \\
\hline & Human & 93 & 15 & 16.13 & 760 & 1.97 \\
\hline
\end{tabular}

The results show that IncRNA transcripts assembled using the RNA-seq libraries of only one species can in many cases be directly mapped to the genome of another species, assuming the IncRNA is located within a region of conserved synteny

downstream of a focal gene), no corresponding assembled lncRNA was annotated in the species used for comparison, although for both species a similar range of tissues was sequenced. For example, of the 2927 syntenic blocks in the sheep/cattle comparison, $347(12 \%)$ of the sheep blocks, and 506 (17\%) of the cattle blocks, contain a lncRNA in the 'upstream' position (that is, between genes 1 and 2), with little overlap between the two species: in only 139 blocks (5\%) is a lncRNA present in this position in both species [see Additional file 3: Table S12]. Similar results are found if the 'downstream' position is considered, as well as the sheep/goat, goat/cattle, sheep/ human, goat/human and cattle/human comparisons: approximately 2 to 5 times as many lncRNAs are found in either of the two species than are found in both [see Additional file 3: Tables S13, S14, S15, S16 and S17].

Each set of syntenic blocks, by definition, represents a set of conserved intergenic regions. Given that the majority of the lncRNAs are intergenic [see Additional file 1: Table S8 and Additional file 2: Table S9], these regions are reasonable locations for mapping candidate transcripts (strictly speaking, concatenated exon sequences) directly to the genome. For the syntenic blocks in each species comparison, we made global alignments of the lncRNAs in species $x$ to the intergenic region of species $y$, and vice versa (see Methods section). Retaining only those alignments in which the lncRNA can match the intergenic region with 20 or more consecutive residues (the majority of these alignments in any case have more than $75 \%$ identity across their entire length), we predicted 1077 additional lncRNAs in cattle, 1401 in sheep, and 1735 in goat, although only 44 in humans (Table 2 ).

The fact that comparatively few ruminant IncRNAs are recognisable at the sequence level in humans (and vice versa) is consistent with the rapid turnover of the lncRNA repertoire between species [92]. In the case of the goat, the number of new IncRNAs predicted by this approach is $50 \%$ more than the number captured (and shortlisted) using goat-specific RNA-seq (Fig. 2).

This suggests that for the purposes of lncRNA detection, datasets from related species can help overcome limitations of sequencing breadth and depth. This is even apparent with comparatively large datasets-the sheep RNA-seq, for instance, spans more tissues and developmental stages than goat, but in absolute terms, it still fails to generate assemblies of many lncRNAs.

\section{Many of the sheep IncRNAs inferred by synteny-which could not be fully assembled from the RNA-seq reads: are nevertheless detectably expressed}

To determine the expression level of the sheep lncRNAs, we used a subset of 71 high-depth ( $>100$ million reads) RNA-seq libraries from the sheep expression atlas [46]. This subset constitutes a set of 11 transcriptionallyrich tissues (bicep muscle, hippocampus, ileum, kidney medulla, left ventricle, liver, ovary, reticulum, spleen, testes, thymus), plus one cell type under two conditions (bone marrow derived macrophages, unstimulated and 


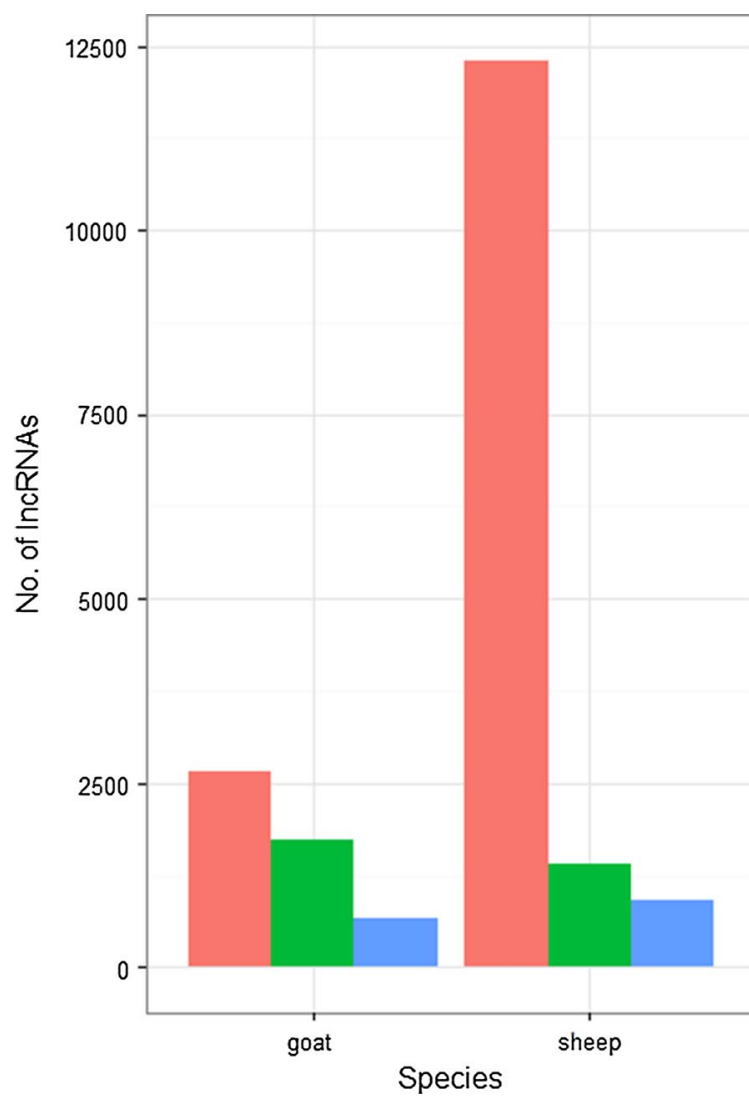

\section{Classification of IncRNA}

Assembled in this species (shortlist)

Mapped from other species

Mapped from other species, and expressed

Fig. 2 Stochastic detection and assembly of IncRNAs by RNA-seq libraries. These results-a consequence of limitations in sequencing breadth and depth—suggest that for a given species, only a subset of the total IncRNA transcriptome is likely to be captured. Nevertheless, the number of candidate IncRNAs for that species can be increased if directly mapping, to a positionally conserved region of the genome, the IncRNAs from either a related (sheep, goat, cattle) or more distant (human) species. Many of these mapped IncRNAs (which could not be completely reconstructed with the RNA-seq libraries of that species) are nevertheless detectably expressed

$7 \mathrm{~h}$ after simulation with lipopolysaccharide), each of which was sequenced in up to six individuals (where possible, three adult males and three adult females).

For each sample, expression was quantified-as transcripts per million (TPM) - using the quantification tool Kallisto [81] [see Additional file 1: Table S19]. Kallisto quantifies expression by matching $\mathrm{k}$-mers from the RNA-seq reads to a pre-built index of $k$-mers, derived from a set of reference transcripts. For sheep, we supplemented the complete set of Oar v3.1 reference transcripts ( $\mathrm{n}=28,828$ transcripts, representing 26,764 genes) both with the shortlist of 11,646 novel lncRNAs (each of which is a single-transcript gene model) [see Additional file 1: Table S1], and those lncRNAs assembled from either human, goat and cattle (respectively, 18, 164 and 1219 lncRNA; see Table 2), the presence of which was predicted in sheep by mapping the transcript to a conserved genomic region.

Of these 13,047 novel lncRNAs, 8826 were detected at a level of TPM higher than 1 in at least one of the 71 adult samples, including 14 of the human transcripts (78\%), 128 of the goat transcripts $(78 \%)$, and 772 of the cattle transcripts (63\%) [see Additional file 1: Table S19]. At a depth of coverage of 100 million reads, we would expect to detect transcripts reproducibly at between 0.01 and 0.1 TPM if they are expressed in all the libraries that are derived from the same tissue/cell type. Indeed, of the 13,047 total novel lncRNAs, 5353 (41\%) were detected with at least one paired-end read in all six replicates of the tissue in which it is most highly expressed [see Additional file 1: Table S19]. Those lncRNAs derived from goat and cattle transcripts are similarly reproducible: 83 (51\%) of the goat transcripts were detected with at least one paired-end read in all 6 replicates of its most expressed tissue, as were $570(47 \%)$ of the cattle transcripts, and 7 (39\%) of the human transcripts [see Additional file 2: Table S20].

By extension, we can consider sheep, cattle and human lncRNAs to be goat lncRNAs, and create a Kallisto index containing candidate lncRNAs that are extracted from 
the goat genome after mapping sheep and cattle transcripts. Using such a Kallisto index (which contains the 2657 shortlisted goat lncRNAs [see Additional file 2: Table S2], 507 sheep lncRNAs, 1213 cattle lncRNAs, and 15 human lncRNAs), 1478 (34\%) of a total set of 4392 candidate goat lncRNAs were reproducibly detected $(>0.01 \mathrm{TPM})$ in all four sampled goats [see Additional file 2: Table S20]. Hence, data from the sheep expression atlas can be used to provide additional functional annotation of the goat genome, in spite of the much smaller number of tissue samples compared to sheep.

In general, lncRNA expression is low: 12,325 sheep lncRNAs (94\% of the total) have a mean TPM less than 10 across all 71 samples. The mean and median maximum TPM for each IncRNA across the total sheep dataset was 18.4 and 2.2 TPM, respectively [see Additional file 1: Table S19]). Other reports have described pervasive, but low-level, mammalian lncRNA transcription [12], and-given that the mean TPM exceeds the median-a high degree of lncRNA tissue-specificity [93-95]. Indeed, for those lncRNAs detected at a TPM less than 1 , the average value of tau-a scalar measure of expression breadth bound between 0 (for housekeeping genes) and 1 (for genes expressed in one sample only) [83] (see Methods section)-is 0.66. Although most of the lncRNAs $(\mathrm{n}=4972,65 \%$ of the 7627 lncRNAs with an average TPM higher than 1 in at least one tissue) have idiosyncratic 'mixed expression' profiles (see Methods section), 1339 lncRNAs (17\%) are nevertheless detected at an average TPM higher than 1 in all 13 tissues [see Additional file 1: Table S19]. Many are enriched in specific tissues, with 905 (12\%) lncRNAs exhibiting either a testes-enriched (that is, fivefold higher expression in testes than other tissues) or testes-specific expression pattern (that is a TPM higher than 1 in the testes and equal to 0 in all other tissues), which is consistent with a previous study that identified numerous lncRNAs involved in ovine testicular development and spermatogenesis [96]. Many lncRNAs are expressed most strongly, even if not uniquely, in the testes. Each gene can be associated with a tissue in which it has the highest preferential expression measure (PEM; see Methods section). For the majority of genes, this tissue is the testes $(n=3770,49 \%$ of the 7627 IncRNA with an average TPM higher than 1 in at least one tissue) [see Additional file 1: Table S19].

\section{Few IncRNAs are fully captured by biological replicates of the same RNA-seq library}

In the largest assembly of predicted lncRNAs, from humans, the transfrags (transcript fragments) assembled from 7256 RNA-seq libraries were consolidated into 58,648 candidate lncRNAs [80]. Before assembling transfrags, machine-learning methods were used to filter, from each library, any library-specific background noise (genomic DNA contamination and incompletely processed RNA). Then, filtered libraries were merged before assembling the final gene models, which in effect is equivalent to pooling together transfrags (which may be partial or full-length transcripts) from all possible libraries. Consequently, a given set of transfrags can be assembled into a consensus transcript for a lncRNA, but that consensus transcript might not actually exist in any one cellular source. The only unequivocal means to confirm the full-length expression would be to clone the full-length cDNA. However, additional confidence could be obtained by increasing the depth of coverage in the same tissue/cell type in a technical replicate. In the sheep expression atlas, 31 diverse tissues/cell types were sampled in each of six individual adults (three females, three males, all unrelated virgin animals approximately 2 years old). By taking a subset of 31 common tissues per individual, each of the six adults was represented by $\sim 0.75$ billion reads.

In a typical lncRNA assembly pipeline, read alignments from all individuals are merged, to maximise the number of candidate gene models (using, for instance, StringTie-merge; see Methods). With $n=6$ adults (and $\sim 0.75$ billion reads per adult), there are $2^{n}-1=63$ possible combinations of data for which GTF can be made with StringTie-merge. The reproducibility of each shortlisted lncRNA, in terms of the number of GTF it is reconstructed in, is shown in Table S21 [see Additional file 1: Table S21]. The GTF themselves are available via the University of Edinburgh DataShare portal; http:// dx.doi.org/10.7488/ds/2284.

Only 812 of the 12,296 sheep lncRNAs (6.6\%) could be fully reconstructed by any of the 63 GTF combinations [see Additional file 1: Table S21]. One caveat in this assessment is that these sheep libraries are exclusively from adults. Many of the 12,296 lncRNA models may instead be expressed during embryonic development. There is evidence of extensive embryonic lncRNA expression in humans $[15,97]$ and mouse $[16,98]$. The lack of embryonic tissues could also explain why fewer lncRNAs were assembled in goat. Nevertheless, when considering all 429 RNA-seq libraries in the sheep expression atlas (i.e. including non-adult samples), there are only, on average, 29 libraries (7\%) in which any individual lncRNA can be fully reconstructed (Fig. 3 and Additional file 1: Table S22).

In many cases, full-length sheep IncRNAs cannot be reconstructed using all the reads sequenced from a given individual. For instance, the known lncRNA ENSOARG00000025201 is reconstructed by 28 of the 63 possible GTF, but none of these GTF was built using reads from only one individual [see Additional file 1: 


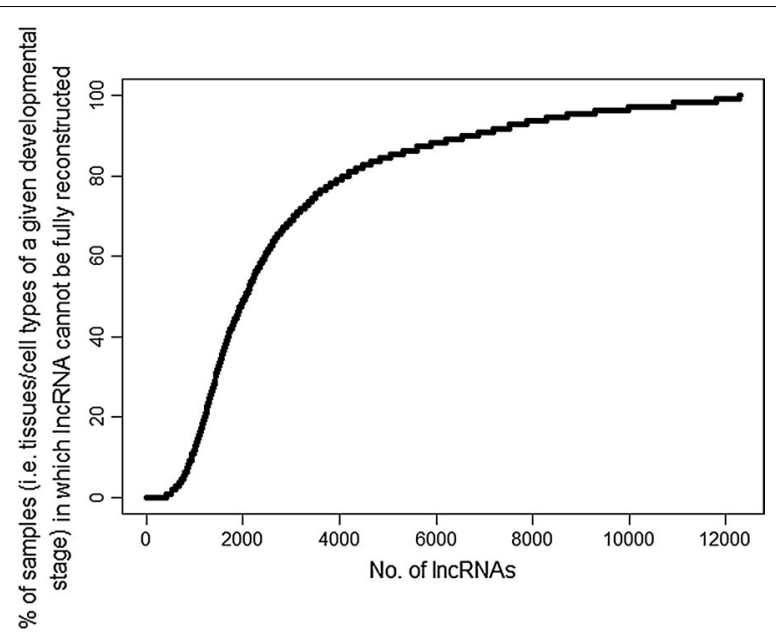

Fig. 3 Proportion of sheep expression atlas samples for which a candidate IncRNA cannot be fully reconstructed. The sheep expression atlas comprises 429 RNA-seq libraries, representing 110 distinct samples; that is, each sample is a tissue/cell type at a given developmental stage, with up to six replicates per sample. Twenty-two candidate IncRNAs cannot be reconstructed in any given sample (i.e., the proportion of samples is 100\%). These IncRNAs could be assembled only after pooling data from multiple samples. Data for this figure are in Additional file 1: Table S22

Table S21]. Only 189 lncRNAs (1.5\%) were fully reconstructed in all 63 possible GTF. Notably, 154 of these are known Ensembl lncRNAs [see Additional file 1: Table S21].

\section{IncRNAs are enriched in the vicinity of co-expressed protein-coding genes}

Enhancer sequences positively modulate the transcription of nearby genes (see reviews $[99,100]$ ), and may be the evolutionary origin of a fraction of these lncRNAs (as suggested by [101, 102]), including a novel class of enhancer-transcribed ncRNAs, enhancer RNAs (eRNAs), which-although a distinct subset-are arbitrarily classified as lncRNAs [103]. eRNAs are likely to be coexpressed with protein-coding genes in their immediate genomic vicinity.

To identify co-regulated sets of protein-coding and non-coding loci, we performed network cluster analysis of the sheep expression level dataset [see Additional file 1: Table S19] using the Markov clustering (MCL) algorithm [87], as implemented by Graphia Professional (Kajeka Ltd., Edinburgh, UK) (see Methods section) [85, 86]. To reduce noise, only those novel lncRNAs with reproducible expression (i.e. that have a TPM higher than 0.01 in every replicate of the tissue in which it is most highly expressed) are included in this analysis $(n=5353)$. The resulting graph contained only genes with tightly correlated expression profiles (Pearson's $r \geq 0.95$ ) (Fig. 4) and was highly structured, organised into clusters of genes with a tissue or cell-type specific expression profile [see Additional file 1: Table S23].

We expect that for a given cluster of co-expressed genes (which contains $x$ lncRNAs and $y$ protein-coding genes, each on chromosome $z$ ), the distance between an enhancer-derived lncRNA and the nearest proteincoding gene should be significantly shorter than the distance between that lncRNA and a random subset of protein-coding genes. For the purposes of this test, each random subset, of size $y$, is drawn from the complete set of protein-coding genes on the same chromosome $z$ (that is, the same chromosome as the lncRNA), irrespective of strand and their degree of co-expression with the lncRNA. The significance of any difference in distance was then assessed using a randomisation test (see Methods section).

Of the 5353 lncRNAs included in the analysis, 1351 (25\%) were found on the same chromosome as a highly co-expressed protein-coding gene [see Additional file 1: Table S24], with 252 of these (19\%) significantly closer to the co-expressed gene than to randomly selected genes from the same chromosome $(p<0.05)$ [see Additional file 1: Table S25].

Even when the lncRNA is reproducibly expressed in each of the six animals, there is still substantial noise in the expression estimates which compromises co-expression analysis. We therefore calculated the Pearson's $r$ correlation between the expression profile of each reproducibly expressed lncRNA and its nearest protein-coding gene (which may overlap it), located both $5^{\prime}$ and $3^{\prime}$ on the sheep genome [see Additional file 1: Table S26]. The distance to the nearest gene correlates negatively with the absolute value of Pearson's $r$, both for genes upstream $\left(r h o=-0.19, p<2.2 \times 10^{-16}\right)$ and downstream $\left(r h o=-0.21, p<2.2 \times 10^{-16}\right)$ of the lncRNA [see Additional file 1: Table S26]. This suggests that, in general, the expression profile of a lncRNA is more similar to nearer than more distant protein-coding genes. Using a variant of the above randomisation test, we also tested whether the absolute value of Pearson's $r$, when correlating the expression profiles of the lncRNA and its nearest proteincoding gene, was significantly greater than the value of $r$ obtained when correlating the lncRNA with 1000 random protein-coding genes drawn from the same chromosome. For this test, analysis was restricted to the lncRNA that were drawn from complete chromosomes rather than the smaller unplaced scaffolds. $27 \%$ of lncRNAs had a Pearson $r$ higher than 0.5 with either the nearest upstream or downstream gene, and in around $20 \%$ of cases, $r$ was significantly different $(p<0.05)$ from the average correlation with the random set [see Additional file 1: Table S26]. 


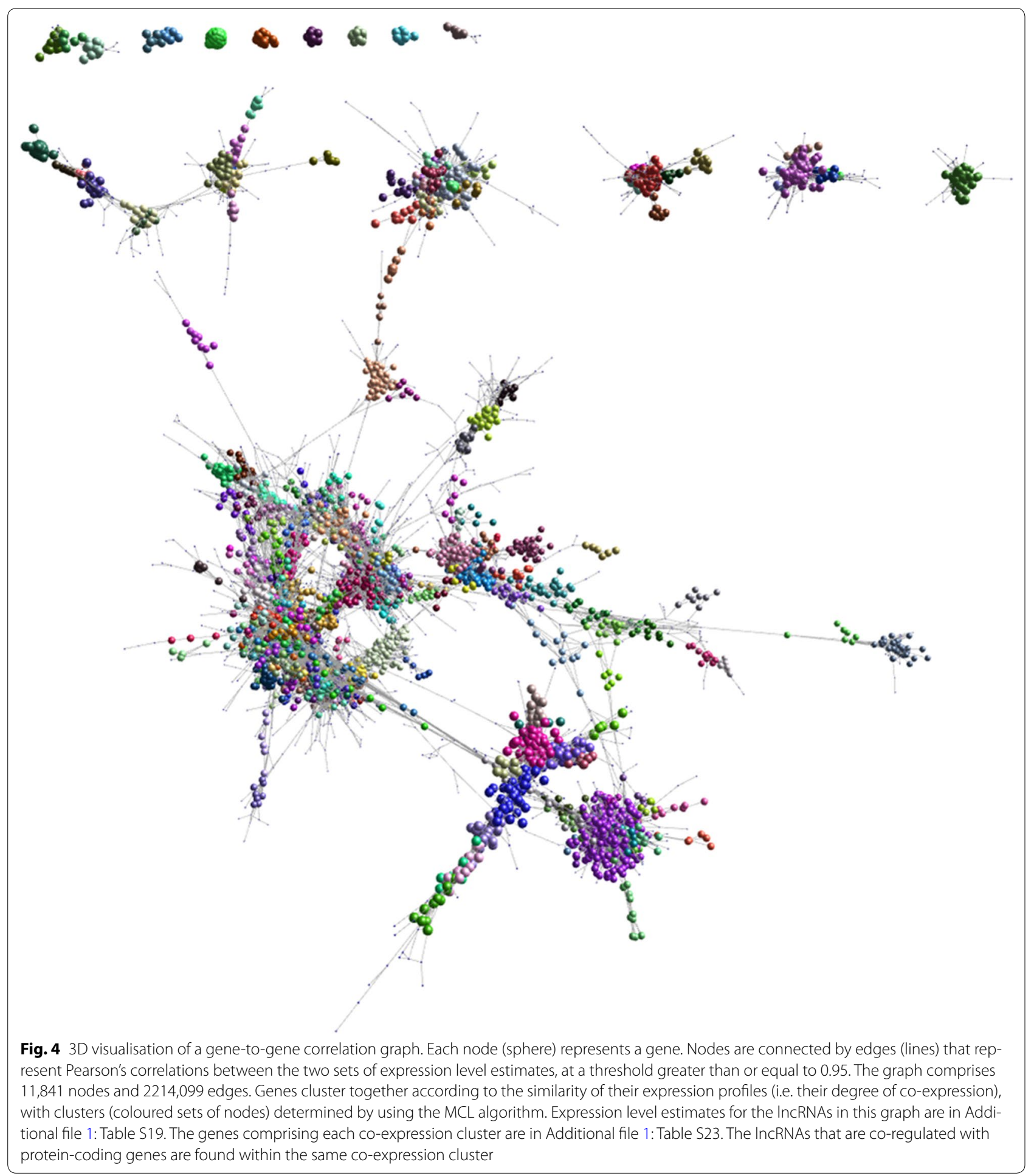

There are, in total, $2 \times 6720 \operatorname{lncRNA}$ /protein-coding gene pairs (i.e., the lncRNA both with its nearest upand nearest downstream gene, excluding 491 lncRNAs on unplaced scaffolds, for which pairing was not possible) $\times 13$ tissues/cells in which the expression of each gene is assessed. Of these 174,720 possible observations, there are 45,959 instances (26\%) in which the lncRNA and the nearest protein-coding gene are both detectably expressed (TPM higher than 1), of which the largest number are found in the testes $(n=2477$ lncRNAs $)$ 
and hippocampus $(\mathrm{n}=2393)$ [see Additional file 1: Table S26]. In conjunction with a generally closer proximity to co-expressed protein-coding genes, this further suggests there is biological relevance for particular lncRNA/ mRNA pairings, particularly in these tissues.

\section{Conclusions}

Comparative analysis of lncRNAs that are assembled using RNA-seq data from several closely related species-sheep, goat and cattle-demonstrates that the de novo assembly of lncRNAs requires very high-depth RNA-seq datasets with a large number of replicates (more than six replicates per sample, each sequencing with many more than 100 million reads). The transcription of many lncRNAs that are identified by this crossspecies approach is conserved, which is a reasonable confirmation of their existence. We identified a subset of lncRNAs in close proximity to protein-coding genes with which they are strongly co-expressed, which is consistent with the evolutionary origin of some ncRNAs in enhancer sequences. Conversely, the majority of lncRNAs are not co-expressed with neighbouring proteincoding genes. Overall, alongside substantially expanding the lncRNA repertoire for several livestock species, we demonstrate that the conventional approach to lncRNA detection-that is, species-specific de novo assembly-can be reliably supplemented by data from related species.

\section{Additional files}

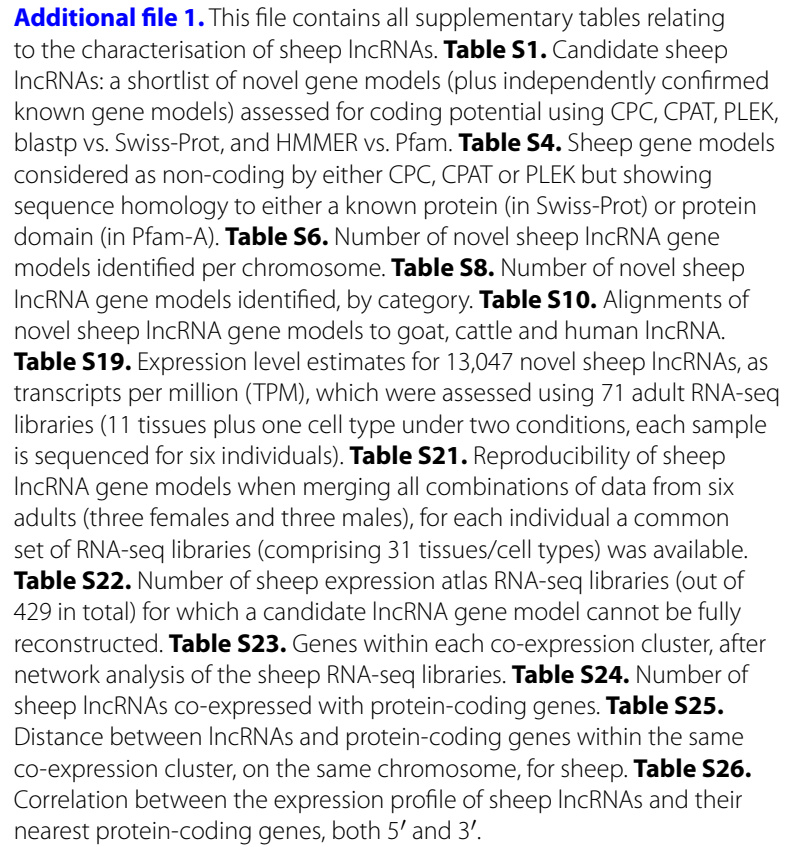

Additional file 2. This file contains all supplementary tables relating to the characterisation of goat IncRNAs. Table S2. Candidate goat IncRNAs: a shortlist of novel gene models assessed for coding potential using CPC, CPAT, PLEK, blastp vs. Swiss-Prot, and HMMER vs. Pfam. Table S5. Goat gene models considered non-coding by either CPC, CPAT or PLEK but showing sequence homology to either a known protein (in Swiss-Prot) or protein domain (in Pfam-A). Table S7. Number of novel goat IncRNA gene models identified per chromosome. Table S9. Number of novel goat IncRNA gene models identified, by category. Table S11. Alignments of novel goat IncRNA gene models to sheep, cattle and human IncRNA. Table S20. Expression level estimates for 4392 novel goat IncRNAs, as transcripts per million (TPM), which were assessed using 54 RNA-seq libraries (20 tissues plus one cell type under two different conditions, for each sample four individuals were sequenced).

Additional file 3. This file contains all supplementary tables relating to IncRNA identification via the conservation of synteny. Table $\mathbf{S 3}$. InCRNAs inferred in one species by the genomic alignment of a transcript assembled with the RNA-seq libraries from a related spdecies. Table S12. Presence of intergenic IncRNAs both in sheep and cattle, in regions of conserved synteny. Table S13. Presence of intergenic IncRNAs both in sheep and goat, in regions of conserved synteny. Table S14. Presence of intergenic IncRNAs both in cattle and goat, in regions of conserved synteny. Table S15. Presence of intergenic IncRNAs both in sheep and humans, in regions of conserved synteny. Table S16. Presence of intergenic IncRNAs both in goat and humans, in regions of conserved synteny. Table S17. Presence of intergenic IncRNAs both in cattle and humans, in regions of conserved synteny. Table $\mathbf{S 1 8}$. High-confidence IncRNA pairs, those conserved across species both sequentially and positionally.

\section{Authors' contributions}

ELC and DAH conceived and coordinated the study. ELC, CM, MEBM and ILF performed the RNA extractions for the sheep and goat samples of the expression atlas and contributed to the experimental planning and design. ELC performed the network cluster analysis. SJB performed all bioinformatic analyses. SJB wrote the manuscript. All authors read and approved the final manuscript.

\section{Author details}

${ }^{1}$ The Roslin Institute, University of Edinburgh, Easter Bush Campus, Edinburgh, Midlothian EH25 9RG, UK. ${ }^{2}$ Nuffield Department of Clinical Medicine, John Radcliffe Hospital, University of Oxford, Headington, Oxford OX3 9DU, UK.

${ }^{3}$ Centre for Synthetic and Systems Biology, $\mathrm{CH}$ Waddington Building, Max Borne Crescent, King's Buildings, University of Edinburgh, Edinburgh EH9 3BF, UK. ${ }^{4}$ Translational Research Institute, Mater Research-University of Queensland, 37 Kent Street, Woolloongabba, QLD 4102, Australia.

\section{Acknowledgements}

The authors would like to thank the farm staff at Dryden farm and members of the sheep tissue collection team from The Roslin Institute and R(D)SVS who were involved in tissue collections for the sheep gene expression atlas project. Rachel Young and Lucas Lefevre isolated the bone marrow derived macrophages and Zofia Lisowski provided technical assistance with collection and post-mortem for the goat samples. Technical expertise for dissection of the sheep brain samples was provided by Fiona Houston and heart samples by Kim Summers and Hiu-Gwen Tsang. The authors are also grateful for the support of the FAANG Data Coordination Centre in the upload and archiving of the sample data and metadata.

\section{Competing interests}

The authors declare they have no competing interests.

\section{Availability of data and materials}

All data analysed during this study are included in this published article and its additional files. The raw RNA-sequencing data are deposited in the European Nucleotide Archive (ENA) under study accessions PRJEB19199 (sheep) and PRJEB23196 (goat). Sample metadata for all tissue and cell samples, prepared 
in accordance with FAANG consortium metadata standards, are deposited in the EBI BioSamples database under group identifiers SAMEG317052 (sheep) and SAMEG330351 (goat). All experimental protocols are available on the FAANG consortium website at http://www.ftp.faang.ebi.ac.uk/ftp/protocols.

\section{Consent for publication}

Not applicable.

\section{Ethics approval and consent to participate}

Approval was obtained from The Roslin Institute's and the University of Edinburgh's Protocols and Ethics Committees. All animal work was carried out under the regulations of the Animals (Scientific Procedures) Act 1986.

\section{Funding}

This work was supported by a Biotechnology and Biological Sciences Research Council (BBSRC; www.bbsrc.ac.uk) grant BB/L001209/1 ('Functional Annotation of the Sheep Genome') and Institute Strategic Program grants 'Farm Animal Genomics'(BBS/E/D/2021550), 'Blueprints for Healthy Animals' (BB/P013732/1) and 'Transcriptomes, Networks and Systems' (BBS/E/D/20211552). The goat RNA-seq data was funded by the Roslin Foundation (www.roslinfoundation. com) which also supported SJB. CM was supported by a Newton Fund Ph.D. studentship (www.newtonfund.ac.uk). Edinburgh Genomics is partly supported through core grants from the BBSRC (BB/J004243/1), National Research Council (NERC; www.nationalacademies.org.uk/nrc) (R8/H10/56), and Medical Research Council (MRC; www.mrc.ac.uk) (MR/K001744/1). The funders had no role in study design, data collection and analysis, decision to publish, or preparation of the manuscript.

\section{Publisher's Note}

Springer Nature remains neutral with regard to jurisdictional claims in published maps and institutional affiliations.

Received: 30 January 2018 Accepted: 13 April 2018 Published online: 24 April 2018

\section{References}

1. Ponting $C P$, Oliver PL, Reik W. Evolution and functions of long noncoding RNAs. Cell. 2009;136:629-41.

2. Engreitz JM, Ollikainen N, Guttman M. Long non-coding RNAs: spatial amplifiers that control nuclear structure and gene expression. Nat Rev Mol Cell Biol. 2016;17:756-70.

3. Rinn JL, Chang HY. Genome regulation by long noncoding RNAs. Ann Rev Biochem. 2012;81:145-66.

4. Chen J, Xue Y. Emerging roles of non-coding RNAs in epigenetic regulation. Sci China Life Sci. 2016;59:227-35.

5. Kung JT, Colognori D, Lee JT. Long noncoding RNAs: past, present, and future. Genetics. 2013;193:651-69.

6. Quinn JJ, Chang HY. Unique features of long non-coding RNA biogenesis and function. Nat Rev Genet. 2016;17:47-62.

7. Villegas VE, Zaphiropoulos PG. Neighboring gene regulation by antisense long non-coding RNAs. Int J Mol Sci. 2015;16:3251-66.

8. Goff LA, Rinn JL. Linking RNA biology to InCRNAs. Genome Res. 2015;25:1456-65.

9. Cech TR, Steitz JA. The noncoding RNA revolution-trashing old rules to forge new ones. Cell. 2014;157:77-94.

10. Kapranov P, St Laurent G, Raz T, Ozsolak F, Reynolds CP, Sorensen PHB, et al. The majority of total nuclear-encoded non-ribosomal RNA in a human cell is 'dark matter' un-annotated RNA. BMC Biol. 2010;8:149.

11. van Bakel H, Nislow C, Blencowe BJ, Hughes TR. Most "dark matter" transcripts are associated with known genes. PLoS Biol. 2010;8:e1000371.

12. Kornienko AE, Dotter CP, Guenzl PM, Gisslinger H, Gisslinger B, Cleary $C$, et al. Long non-coding RNAs display higher natural expression variation than protein-coding genes in healthy humans. Genome Biol. 2016;17:14.

13. Mercer TR, Dinger ME, Sunkin SM, Mehler MF, Mattick JS. Specific expression of long noncoding RNAs in the mouse brain. Proc Nat Acad Sci USA. 2008;105:716-21.
14. Gloss BS, Dinger ME. The specificity of long noncoding RNA expression. Biochim Biophys Acta. 2016;1859:16-22.

15. Qiu JJ, Ren ZR, Yan JB. Identification and functional analysis of long noncoding RNAs in human and mouse early embryos based on single-cell transcriptome data. Oncotarget. 2016;7:61215-28.

16. Zhang K, Huang K, Luo Y, Li S. Identification and functional analysis of long non-coding RNAs in mouse cleavage stage embryonic development based on single cell transcriptome data. BMC Genomics. 2014;15:845.

17. Pauli A, Valen E, Lin MF, Garber M, Vastenhouw NL, Levin JZ, et al. Systematic identification of long noncoding RNAs expressed during zebrafish embryogenesis. Genome Res. 2012;22:577-91.

18. Cabili MN, Trapnell C, Goff L, Koziol M, Tazon-Vega B, Regev A, et al. Integrative annotation of human large intergenic noncoding RNAs reveals global properties and specific subclasses. Genes Dev. 2011;25:1915-27.

19. Johnsson P, Lipovich L, Grander D, Morris KV. Evolutionary conservation of long non-coding RNAs; sequence, structure, function. Biochim Biophys Acta. 2014;1840:1063-71.

20. Andersson R, Refsing Andersen P, Valen E, Core LJ, Bornholdt J, Boyd $M$, et al. Nuclear stability and transcriptional directionality separate functionally distinct RNA species. Nat Commun. 2014;5:5336.

21. Jia H, Osak M, Bogu GK, Stanton LW, Johnson R, Lipovich L. Genomewide computational identification and manual annotation of human long noncoding RNA genes. RNA. 2010;16:1478-87.

22. Maeda N, Kasukawa T, Oyama R, Gough J, Frith M, Engstrom PG, et al. Transcript annotation in FANTOM3: mouse gene catalog based on physical cDNAs. PLoS Genet. 2006;2:e62.

23. Sasaki YT, Sano M, Ideue T, Kin T, Asai K, Hirose T. Identification and characterization of human non-coding RNAs with tissue-specific expression. Biochem Biophys Res Commun. 2007;357:991-6.

24. Ravasi T, Suzuki H, Pang KC, Katayama S, Furuno M, Okunishi R, et al. Experimental validation of the regulated expression of large numbers of non-coding RNAs from the mouse genome. Genome Res. 2006;16:11-9.

25. Katayama S, Tomaru Y, Kasukawa T, Waki K, Nakanishi M, Nakamura M, et al. Antisense transcription in the mammalian transcriptome. Science. 2005;309:1564-6.

26. Mattick JS, Rinn JL. Discovery and annotation of long noncoding RNAs. Nat Struct Mol Biol. 2015;22:5-7.

27. Balwierz PJ, Carninci P, Daub CO, Kawai J, Hayashizaki Y, Van Belle W, et al. Methods for analyzing deep sequencing expression data: constructing the human and mouse promoterome with deepCAGE data. Genome Biol. 2009;10:R79.

28. McIntyre LM, Lopiano KK, Morse AM, Amin V, Oberg AL, Young LJ, et al. RNA-seq: technical variability and sampling. BMC Genomics. 2011;12:293.

29. Steijger T, Abril JF, Engstrom PG, Kokocinski F, Hubbard TJ, Guigo R, et al. Assessment of transcript reconstruction methods for RNA-seq. Nat Methods. 2013;10:1177-84.

30. Hon CC, Ramilowski JA, Harshbarger J, Bertin N, Rackham OJL, Gough J, et al. An atlas of human long non-coding RNAs with accurate $5^{\prime}$ ends. Nature. 2017;543:199-204.

31. Koufariotis LT, Chen YP, Chamberlain A, Vander Jagt C, Hayes BJ. A catalogue of novel bovine long noncoding RNA across 18 tissues. PLoS One. 2015:10:e0141225.

32. Zhou ZY, Li AM, Adeola AC, Liu YH, Irwin DM, Xie HB, et al. Genomewide identification of long intergenic noncoding RNA genes and their potential association with domestication in pigs. Genome Biol Evol. 2014;6:1387-92.

33. Scott EY, Mansour T, Bellone RR, Brown CT, Mienaltowski MJ, Penedo $\mathrm{MC}$, et al. Identification of long non-coding RNA in the horse transcriptome. BMC Genomics. 2017;18:511.

34. Bakhtiarizadeh MR, Hosseinpour B, Arefnezhad B, Shamabadi N, Salami SA. In silico prediction of long intergenic non-coding RNAs in sheep. Genome. 2016;59:263-75.

35. Billerey C, Boussaha M, Esquerré D, Rebours E, Djari A, Meersseman C, et al. Identification of large intergenic non-coding RNAs in bovine muscle using next-generation transcriptomic sequencing. BMC Genomics. 2014;15:499. 
36. Liu XF, Ding XB, Li X, Jin CF, Yue YW, Li GP, et al. An atlas and analysis of bovine skeletal muscle long noncoding RNAs. Anim Genet. 2017:48:278-86.

37. Weikard R, Hadlich F. Kuehn C. Identification of novel transcripts and noncoding RNAs in bovine skin by deep next generation sequencing. BMC Genomics. 2013;14:789.

38. Yu L, Tai L, Zhang L, Chu Y, Li Y, Zhou L. Comparative analyses of long non-coding RNA in lean and obese pig. Oncotarget. 2017;8:41440-50.

39. Wang J, Hua L, Chen J, Zhang J, Bai X, Gao B, et al. Identification and characterization of long non-coding RNAs in subcutaneous adipose tissue from castrated and intact full-sib pair Huainan male pigs. BMC Genomics. 2017;18:542.

40. Xia J, Xin L, Zhu W, Li L, Li C, Wang Y, et al. Characterization of long noncoding RNA transcriptome in high-energy diet induced nonalcoholic steatohepatitis minipigs. Sci Rep. 2016;6:30709.

41. Esteve-Codina A, Kofler R, Palmieri N, Bussotti G, Notredame C, PérezEnciso M. Exploring the gonad transcriptome of two extreme male pigs with RNA-seq. BMC Genomics. 2011:12:552

42. Engreitz JM, Haines JE, Perez EM, Munson G, Chen J, Kane M, et al. Local regulation of gene expression by IncRNA promoters, transcription and splicing. Nature. 2016;539:452-5.

43. Derrien T, Johnson R, Bussotti G, Tanzer A, Djebali S, Tilgner H, et al. The GENCODE v7 catalog of human long noncoding RNAs: analysis of their gene structure, evolution, and expression. Genome Res. 2012:22:1775-89.

44. Carninci P, Kasukawa T, Katayama S, Gough J, Frith MC, Maeda N, et al. The transcriptional landscape of the mammalian genome. Science. 2005;309:1559-63.

45. Roux BT, Heward JA, Donnelly LE, Jones SW, Lindsay MA. Catalog of differentially expressed long non-coding RNA following activation of human and mouse innate immune response. Front Immunol. 2017:8:1038

46. Clark EL, Bush SJ, McCulloch MEB, Farquhar IL, Young R, Lefevre L, et al. A high resolution atlas of gene expression in the domestic sheep (Ovis aries). PLoS Genet. 2017:13:e1006997.

47. Kumar S, Stecher G, Suleski M, Hedges SB. TimeTree: a resource for timelines, timetrees, and divergence times. Mol Biol Evol. 2017:34:1812-9.

48. Weikard R, Demasius W, Kuehn C. Mining long noncoding RNA in livestock. Anim Genet. 2017;48:3-18.

49. Andersson L, Archibald AL, Bottema CD, Brauning R, Burgess SC, Burt $D W$, et al. Coordinated international action to accelerate genome-tophenome with FAANG, the functional annotation of animal genomes project. Genome Biol. 2015;16:57.

50. Tuggle CK, Giuffra E, White SN, Clarke L, Zhou H, Ross PJ, et al. GOFAANG meeting: a gathering on functional annotation of animal genomes. Anim Genet. 2016:47:528-33.

51. Su Al, Cooke MP, Ching KA, Hakak Y, Walker JR, Wiltshire T, et al. Largescale analysis of the human and mouse transcriptomes. Proc Nat Acad Sci USA. 2002;99:4465-70.

52. Su Al, Wiltshire T, Batalov S, Lapp H, Ching KA, Block D, et al. A gene atlas of the mouse and human protein-encoding transcriptomes. Proc Nat Acad Sci USA. 2004;101:6062-7.

53. Gautier EL, Shay T, Miller J, Greter M, Jakubzick C, Ivanov S, et al. Gene expression profiles and transcriptional regulatory pathways underlying mouse tissue macrophage identity and diversity. Nat Immunol. 2012;13:1118-28.

54. Kim D, Langmead B, Salzberg SL. HISAT: a fast spliced aligner with low memory requirements. Nat Methods. 2015;12:357-60.

55. Pertea M, Pertea GM, Antonescu CM, Chang TC, Mendell JT, Salzberg SL. StringTie enables improved reconstruction of a transcriptome from RNA-seq reads. Nat Biotechnol. 2015;33:290-5.

56. Ilott NE, Ponting CP. Predicting long non-coding RNAs using RNA sequencing. Methods. 2013;63:50-9.

57. Bickhart DM, Rosen BD, Koren S, Sayre BL, Hastie AR, Chan S, et al. Single-molecule sequencing and chromatin conformation capture enable de novo reference assembly of the domestic goat genome. Nat Genet. 2017;49:643-50.

58. Slater GSC, Birney E. Automated generation of heuristics for biological sequence comparison. BMC Bioinformatics. 2005;6:31.
59. Camacho C, Coulouris G, Avagyan V, Ma N, Papadopoulos J, Bealer $\mathrm{K}$, et al. BLAST+: architecture and applications. BMC Bioinformatics. 2009;10:421.

60. Haas BJ, Salzberg SL, Zhu W, Pertea M, Allen JE, Orvis J, et al. Automated eukaryotic gene structure annotation using EVidenceModeler and the program to assemble spliced alignments. Genome Biol. 2008;9:R7.

61. Wang L, Park HJ, Dasari S, Wang S, Kocher JP, Li W. CPAT: coding-potential assessment tool using an alignment-free logistic regression model. Nucleic Acids Res. 2013:41:e74.

62. Fickett JW. Recognition of protein coding regions in DNA sequences. Nucleic Acids Res. 1982;10:5303-18.

63. Fickett JW, Tung CS. Assessment of protein coding measures. Nucleic Acids Res. 1992;20:6441-50.

64. Li A, Zhang J, Zhou Z. PLEK: a tool for predicting long non-coding RNAs and messenger RNAs based on an improved k-mer scheme. BMC Bioinformatics. 2014;15:311.

65. Kong L, Zhang Y, Ye ZQ, Liu XQ, Zhao SQ, Wei L, et al. CPC: assess the protein-coding potential of transcripts using sequence features and support vector machine. Nucleic Acids Res. 2007;35:W345-9.

66. Suzek BE, Huang H, McGarvey P, Mazumder R, Wu CH. UniRef: comprehensive and non-redundant UniProt reference clusters. Bioinformatics. 2007;23:1282-8

67. Suzek BE, Wang Y, Huang H, McGarvey PB, Wu CH. UniRef clusters: a comprehensive and scalable alternative for improving sequence similarity searches. Bioinformatics. 2015;31:926-32.

68. Sing T, Sander O, Beerenwinkel N, Lengauer T. ROCR: visualizing classifier performance in R. Bioinformatics. 2005;21:3940-1.

69. Mistry J, Finn RD, Eddy SR, Bateman A, Punta M. Challenges in homology search: hMMER3 and convergent evolution of coiled-coil regions. Nucleic Acids Res. 2013;41:e121

70. Finn RD, Coggill P, Eberhardt RY, Eddy SR, Mistry J, Mitchell AL, et al. The Pfam protein families database: towards a more sustainable future. Nucleic Acids Res. 2016:44:D279-85.

71. Boutet E, Lieberherr D, Tognolli M, Schneider M, Bairoch A. UniProtKB/ Swiss-Prot. Methods Mol Biol. 2007:406:89-112.

72. UniProt Consortium. UniProt: a hub for protein information. Nucleic Acids Res. 2015:43:D204-12

73. Kinsella RJ, Kahari A, Haider S, Zamora J, Proctor G, Spudich G, et al. Ensembl BioMarts: a hub for data retrieval across taxonomic space. Database (Oxford). 2011:2011:bar030.

74. Ma L, Bajic VB, Zhang Z. On the classification of long non-coding RNAs. RNA Biol. 2013;10:925-33.

75. Zhao Y, Li H, Fang S, Kang Y, Wu W, Hao Y, et al. NONCODE 2016: an informative and valuable data source of long non-coding RNAs. Nucleic Acids Res. 2016;44:D203-8.

76. Quek XC, Thomson DW, Maag JL, Bartonicek N, Signal B, Clark MB, et al. IncRNAdb v.2.0: expanding the reference database for functional long noncoding RNAs. Nucleic Acids Res. 2015;43:D168-73.

77. Rice P, Longden I, Bleasby A. EMBOSS: the European molecular biology open software suite. Trends Genet. 2000;16:276-7.

78. Takahashi H, Kato S, Murata M, Carninci P. CAGE (cap analysis of gene expression): a protocol for the detection of promoter and transcriptional networks. Methods Mol Biol. 2012:786:181-200.

79. Sun L, Luo H, Bu D, Zhao G, Yu K, Zhang C, et al. Utilizing sequence intrinsic composition to classify protein-coding and long non-coding transcripts. Nucleic Acids Res. 2013;41:e166.

80. Iyer MK, Niknafs YS, Malik R, Singhal U, Sahu A, Hosono Y, et al. The landscape of long noncoding RNAs in the human transcriptome. Nat Genet. 2015;47:199-208.

81. Bray NL, Pimentel H, Melsted P, Pachter L. Near-optimal probabilistic RNA-seq quantification. Nat Biotechnol. 2016;34:525-7.

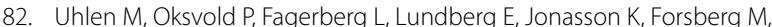
et al. Towards a knowledge-based human protein atlas. Nat Biotechnol. 2010;28:1248-50.

83. Yanai I, Benjamin H, Shmoish M, Chalifa-Caspi V, Shklar M, Ophir R, et al. Genome-wide midrange transcription profiles reveal expression level relationships in human tissue specification. Bioinformatics. 2005:21:650-9. 
84. Huminiecki L, Lloyd A, Wolfe K. Congruence of tissue expression profiles from gene expression atlas. SAGEmap and Tissuelnfo databases. BMC Genomics. 2003;4:31.

85. Freeman TC, Goldovsky L, Brosch M, van Dongen S, Maziere P, Grocock $\mathrm{RJ}$, et al. Construction, visualisation, and clustering of transcription networks from microarray expression data. PLoS Comput Biol. 2007:3:2032-42.

86. Theocharidis A, van Dongen S, Enright AJ, Freeman TC. Network visualization and analysis of gene expression data using BioLayout express(3D). Nat Protoc. 2009;4:1535-50.

87. van Dongen $\mathrm{S}$, Abreu-Goodger C. Using MCL to extract clusters from networks. Methods Mol Biol. 2012;804:281-95.

88. Bush SJ, Castillo-Morales A, Tovar-Corona JM, Chen L, Kover PX, Urrutia AO. Presence-absence variation in A. thaliana is primarily associated with genomic signatures consistent with relaxed selective constraints. Mol Biol Evol. 2014;31:59-69.

89. Liu SJ, Nowakowski TJ, Pollen AA, Lui JH, Horlbeck MA, Attenello FJ, et al. Single-cell analysis of long non-coding RNAs in the developing human neocortex. Genome Biol. 2016;17:67.

90. Chodroff RA, Goodstadt L, Sirey TM, Oliver PL, Davies KE, Green ED, et al. Long noncoding RNA genes: conservation of sequence and brain expression among diverse amniotes. Genome Biol. 2010;11:R72.

91. Bajic VB, Tan SL, Christoffels A, Schonbach C, Lipovich L, Yang L, et al. Mice and men: their promoter properties. PLoS Genet. 2006;2:e54

92. Necsulea A, Soumillon M, Warnefors M, Liechti A, Daish T, Zeller U, et al. The evolution of IncRNA repertoires and expression patterns in tetrapods. Nature. 2014;505:635-40.

93. Tsoi LC, lyer MK, Stuart PE, Swindell WR, Gudjonsson JE, Tejasvi T, et al. Analysis of long non-coding RNAs highlights tissue-specific expression patterns and epigenetic profiles in normal and psoriatic skin. Genome Biol. 2015;16:24.
94. Jiang C, Li Y, Zhao Z, Lu J, Chen H, Ding N, et al. Identifying and functionally characterizing tissue-specific and ubiquitously expressed human IncRNAs. Oncotarget. 2016;7:7120-33.

95. Wu W, Wagner EK, Hao Y, Rao X, Dai H, Han J, et al. Tissue-specific coexpression of long non-coding and coding RNAs associated with breast cancer. Sci Rep. 2016;6:32731.

96. Zhang Y, Yang H, Han L, Li F, Zhang T, Pang J, et al. Long noncoding RNA expression profile changes associated with dietary energy in the sheep testis during sexual maturation. Sci Rep. 2017;7:5180.

97. Bouckenheimer J, Assou S, Riquier S, Hou C, Philippe N, Sansac C, et al. Long non-coding RNAs in human early embryonic development and their potential in ART. Hum Reprod Update. 2016;23:19-40.

98. Karlic R, Ganesh S, Franke V, Svobodova E, Urbanova J, Suzuki Y, et al. Long non-coding RNA exchange during the oocyte-to-embryo transition in mice. DNA Res. 2017;24:129-41.

99. Li W, Notani D, Rosenfeld MG. Enhancers as non-coding RNA transcription units: recent insights and future perspectives. Nat Rev Genet. 2016;17:207-23.

100. Chen H, Du G, Song X, Li L. Non-coding transcripts from enhancers: new insights into enhancer activity and gene expression regulation. Genomics Proteomics Bioinformatics. 2017;15:201-7.

101. De Santa F, Barozzi I, Mietton F, Ghisletti S, Polletti S, Tusi BK, et al. A large fraction of extragenic RNA Pol II transcription sites overlap enhancers. PLoS Biol. 2010;8:e1000384.

102. Kim TK, Hemberg M, Gray JM, Costa AM, Bear DM, Wu J, et al. Widespread transcription at neuronal activity-regulated enhancers. Nature. 2010;465:182-7.

103. Natoli G, Andrau JC. Noncoding transcription at enhancers: general principles and functional models. Annu Rev Genet. 2012;46:1-19.
Ready to submit your research? Choose BMC and benefit from:

- fast, convenient online submission

- thorough peer review by experienced researchers in your field

- rapid publication on acceptance

- support for research data, including large and complex data types

- gold Open Access which fosters wider collaboration and increased citations

- maximum visibility for your research: over 100M website views per year

At BMC, research is always in progress.

Learn more biomedcentral.com/submissions 